\title{
Modern Concepts for Teeth Whitening
}

\author{
Chiara Vagge* \\ Dental Hygienist, Università Vita Salute San Raffaele (Milano), Italy \\ *Corresponding Author: Chiara Vagge, Dental Hygienist, Università Vita Salute San Raffaele (Milano), Italy.
}

Received: August 14, 2019; Published: September 26, 2019

DOI: $10.31080 /$ ASDS.2019.03.0653

\begin{abstract}
A growing number of oral care products focus on teeth whitening. Also only one discolored tooth represent an important aesthetic problem and today it's considered a psychological and social value. For this reason the tooth whitening is the most requested aesthetic intervention. Bleaching offers a minimally invasive aesthetic treatment, with a very low percentage of side effects. The success of bleaching derives from a lot of factors such as the choice of appropriate techniques and treatment planning, a careful diagnosis and the patient's motivation and education. The purpose of this work is to explain the bleaching techniques, the type of treatment, the side effects and the contraindication, explain the dyschromia and social effects.
\end{abstract}

Keywords: Oral Care; Bleaching; Teeth Whitening

\section{Introduction}

The term "bleaching", literally means "lightening", outlines the specific treatment that aims at the brightening of the chroma of the tooth. Today the professional bleaching session is one of the most requested and popular cosmetic treatment in dental offices. It's required both for need and for fashion trend. The natural white of tooth incorporates shades from yellow-brown to gray- blue, with accentuated interpersonal variability. In most cases the yellowbrown tones achieve greater results during tooth whitening than the gray-blue one. There is no predefined or standard color of the chroma, but it varies from subject to subject and from tooth to tooth; for example, in most cases, the canines tend to be darker than the other teeth. Moreover the permanent dentition tends to assume a coloration on the shades of ivory, and the deciduous dentition is more whiter because of the stronger mineralization.

Contrarily to what everybody thinks the color is not defined by the enamel but rather by the underlying dentin. In fact the enamel is translucent. To identify the shade of tooth, dental hygienist use the Vita shade guide. It's illustrated in the figure 1 and it's composed of different shade of colors where B1 represents the whitest tonality. Moreover each colors of the Vita Shade guide differs from subject to subject.

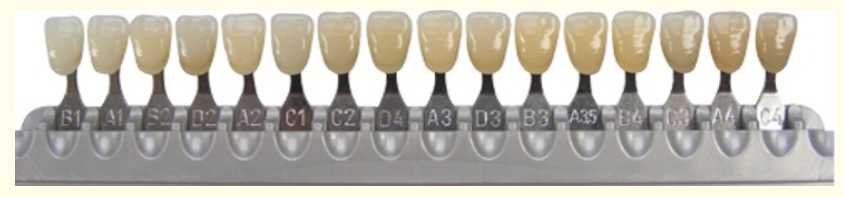

Figure 1: Vita Shade guide.

We defined dyschromia as the not-homogeneous color of the dentin or the different coloration from teeth to teeth (Figure 2.). The dyschromias is present in the majority of the general population and it's etiology can be intrinsic, extrinsic or booth. The first include the intake of drugs for long or short periods (tetracycline, etc.), hyper-fluorosis, food deficiencies (vitamin intake), trauma, bleeding and aging of dental tissues. The second include eating habits (sugary drinks, coffee, tea, wine, cola, fruit juices, etc.), tobacco and thinning of the enamel. 


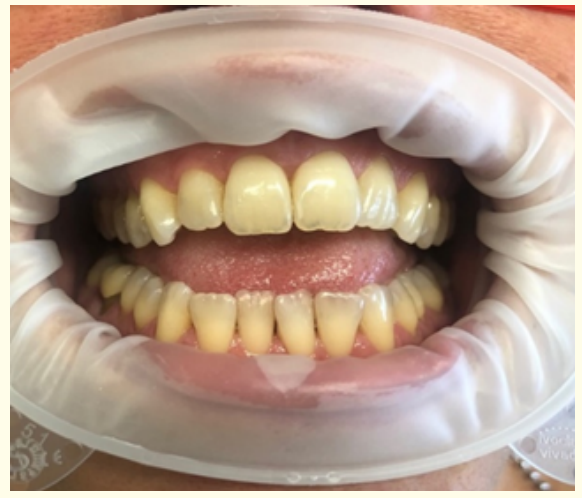

Figure 2: Dyschromias

\section{How does it work}

During a session of bleaching it's applied a substance based on hydrogen peroxide $\left(\mathrm{H}_{2} \mathrm{O}_{2}\right)$ on the surface of the tooth. The gel, after the activation from chemical substances or with a light source, releases reactive oxygen species (ROS). ROS's migrate through the prisms of the enamel and reach the chromogenic molecules, responsible for the tooth yellowing, triggering redox reactions that making it colorless.

Before to start the tooth whitening is mandatory to exclude contraindications at this treatment. The dental hygienist must reconstruct the patient's anamnesis in order to know his habits, carry out an accurate intra and extra oral examination, evaluate and check the presence of restorations, assess the state of tissues and oral mucosa, make if possible a radiographic diagnosis, perform a test for hypersensitivity and underline the importance of a professional oral hygiene session. Only when the previous information's have been collected, the dental hygienist defines the initial tonality of the tooth, through the use of the life scale, and takes the photographic documentation of the patient's mouth. Then he instructs the patient to maintain the result of the bleaching through a "white" diet, the absence of tobacco and substance based on Chlorhexidine intake for at least one week and to maintain regular sessions of professional oral hygiene every 6 - 12 months. Nowadays you can find toothpastes and whitening pens which can help to maintain bleaching; these products can only be purchased at dental offices.

Depending on the severity of the case and the needs of the patient, the dental hygienist will decide whether to propose a home therapy or a treatment performed in the dental office. Both systems use the same chemical agents (oxygen peroxide, carbamide peroxide, sodium perborate and derivatives) with different concentrations. Home bleaching uses masks (universal or customized) that can be combined with syringes containing whitening gel at low concentration; this treatment is called "soft bleaching". The tooth whitening in the dental office allows the dental hygienist to use the active substances at higher concentrations, getting a greater efficacy. For this reason this treatment it is also called "power bleaching".

The permanence of the result obtained with a home bleaching is shorter compared to a "power bleaching", which has a duration of 4 - 6 years. It's important to remember that the subjective variables and habits (even flawed ones) of the patients can compromise the duration of the result.

\section{Contraindications and side effects}

Despite what a lot of people think, dental bleaching does not damage tooth enamel. The last generation products used for bleaching contain Potassium and Fluorine Nitrate. Both of these molecules are recognized for their reinforcing and filling effect on the dental element. Moreover, they help to counteract dental hypersensitivity. The directive 2011/84/EU differentiates the cosmetic dental treatment from the medical one. The products used in the cosmetic treatments have a percentage of $\mathrm{H}_{2} \mathrm{O}_{2}$ that varies from $0.1 \%$ to $6 \%$; the first application is performed and shown in the dental office, then the patient can apply it at home. The medical products have a percentage of $\mathrm{H}_{2} \mathrm{O}_{2}$ that is higher than $6 \%$; for this reason its use is limited to the dental office. The contraindications that the dental hygienist must consider before performing a whitening treatment are dentinal sensitivity, devitalized and reconstructed dental elements, dental supplies, chronic severe pathologies, pregnancy and breastfeeding and presence of caries, gingivitis, pulpits and tartar.

Although they are very some risks associated with bleaching are reported. The most frequent side effects are dentin sensitivity and gingival inflammation. After performing a session of oral hygiene and in anticipation of a session of bleaching, the desensitizing therapy is recommended, using special toothpastes, potassium nitrate masks or topical Fluor prophylaxis. Gingival inflammation is a chemical damage usually temporary and with spontaneous resolution, due to the accidental contact of the activated gel for bleaching with gum; the affected areas can appear white and the 
sensation that the patient can feel is burning. Today there are products with higher concentrations of vitamin C - E, extracts of herbs and propolis that facilitate the healing of inflammation, lesions or burns.

To further reduce the slightest possibility of these side effects there is passive and active prevention. The passive prevention consists in limiting the exposure time with the bleaching gel, in reducing the frequency and the percentage of concentration of $\mathrm{H}_{2} \mathrm{O}_{2}$ and in delaying the time of treatment. For active prevention we mean the desensitization therapy and the elimination from the diet of hot food and cold and hypertonic drinks. The composites and the cladding materials don't meet chromatic variations with the bleaching. An increased mercury emission of the amalgam dental supplies is reported. For this reason is necessary cover the amalgam with a specific lacquer before bleaching.

\section{Critical attributions for the choice of whitening products}

The dental hygienist updates himself about products used nowadays for the bleaching. Before buying a product the dental hygienist must consider: the neutral $\mathrm{pH}$, the water and the desensitizing content, the viscosity, the instruction booklet that must be clear and simple and the scientific value of the product. At last the whitening treatment of vital teeth can be performed either by the dentist and the dental hygienist, but the whitening of nonvital teeth is purely responsibility of the dentist.

\section{Photoactive and not-photo bleaching}

Medical bleaching treatments can be performed with or without a professional lamp. These two methods have completely different results and effects, in fact many scientific studies [...] assert that the photoactivated bleaching has a fairly high scientific value to be defined more effective compared to bleaching not photoactive. More scientific studies [...] have showed that the photoactive bleaching possesses a higher possibility of causing side effects.

For the first clinical case (Figure 3) has been used a photoactive bleaching. The lamp used for the treatment is produced in accordance with EEC Directive 93/42 for medical devices. The lamp operating voltage is $100-240 \mathrm{~V} / 50-60 \mathrm{~Hz}$; the light source with modules has 10 LEDs $x 5 \mathrm{~W}=50 \mathrm{~W}$ and has a light intensity of over $600 \mathrm{~mW} / \mathrm{cm}^{2}$ at $1 \mathrm{~cm}$ distance from the window of polycarbonate. The product used for bleaching does not require a gingival barrier since it has a low concentration of carbamide peroxide equal to $16 \%$ and a low percentage of hydrogen peroxide released equal to $5.6 \%$. The procedure includes three applications; each one has the duration of eight minutes.
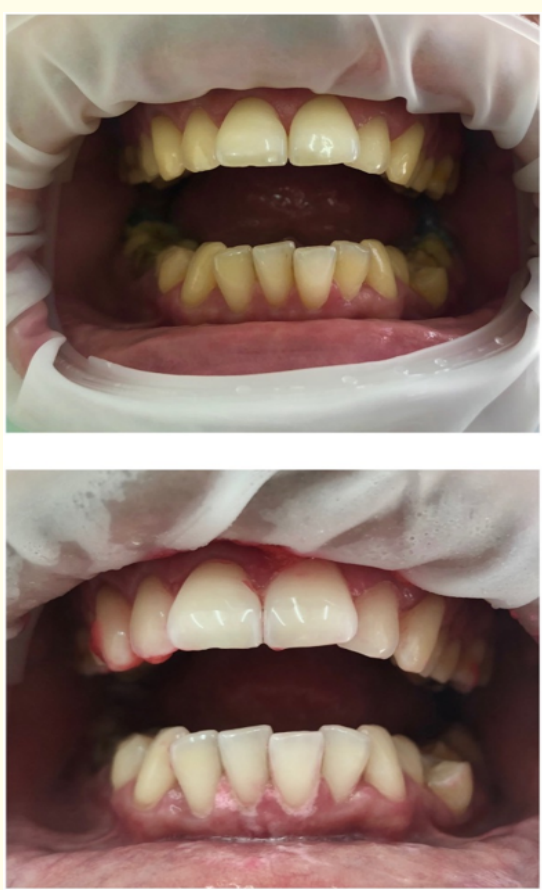

Figure 3: Starting tone: D4, arrival tone: B2.

For the second clinical case (Figure 4) has been used a nonphotoactive bleaching obtained with chemical activation without lamp. For this type of tooth whitening is necessary a gingival barrier. The product contains hydrogen peroxide equal to $40 \%$ and Potassium Nitrate and Fluoride to counteract the unpleasant effects that may arise (with a very low percentage) in the post treatment, such as dentinal hypersensitivity. Moreover, the $\mathrm{pH}$ is neutral. The procedure consists of two applications; each one has the duration of twenty minutes. After the treatment the patient didn't demonstrate any symptom of dentin sensitivity [1-9].

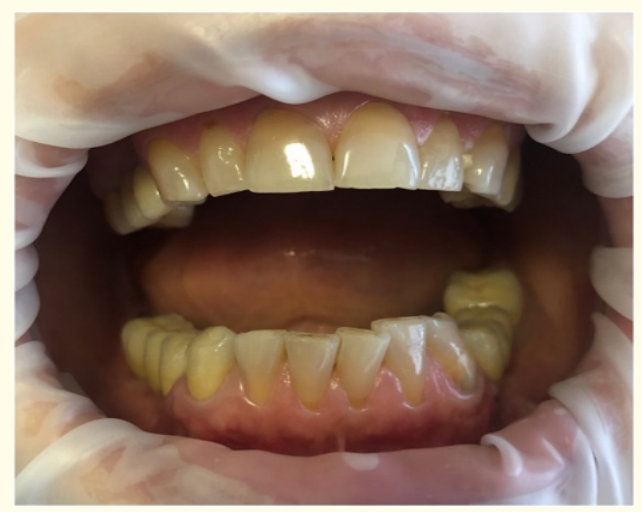




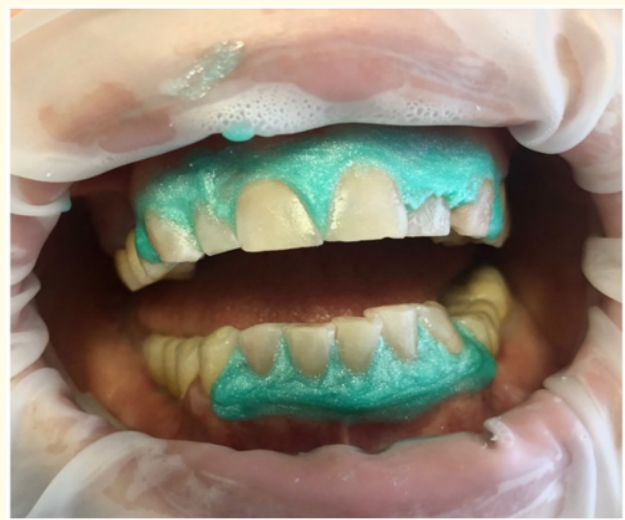

Figure 4: Starting tone: B3, arrival tone: D2.

\section{Conclusion}

In conclusion there are many types of whitening with completely different results and effects. Many works that the photoactivated bleaching has a fairly high scientific value to be defined more effective compared to photoactive. There isn't a standard result, because the bleaching varies from subject to subject. The hygienist updates himself about products used nowadays for the bleaching like: toothpastes, whitening pens to diets. Before buying a product the dental hygienist must consider: the neutral $\mathrm{pH}$, the water and the icing content, the viscosity, the instruction booklet that must be clear and simple and the scientific value of duct. At last to further reduce the slightest possibility of these side effects dental hygienist can do a passive ye prevention. We can define bleaching a safe treatment with important social value today.

\section{Bibliography}

1. Kugel., et al. "Clinical evaluation of chemical and lightactivated tooth whitening systems". Compendium of Continuing Education In Dentistry 27.1 (2006): 54-62.

2. Wiegand., et al. "Licht und Laser oder doch lieber Schiene oder Pinsel?" Schweiz Monatsschr Zahnmed (2006).

3. Buchalla Attin., et al. "External bleaching therapy with activation by heat, light or laser -A systematic review". Dental Materials 23.5 (2007): 586-596.

4. Matthias Epple., et al. Review: A Critical Review of Modern Concepts for Teeth Whitening (2019).
5. Martini., et al. "Does the Use of Reservoirs Have Any Impact on the Efficacy of At-Home Bleaching? A Systematic Review". Brazilian Dental Journal 30.3 (2019): 285-294.

6. Greenwall-Cohen. "The single discoloured tooth: vital and non-vital bleaching techniques". British Dental Journal 226.11 (2019): 839-849.

7. Bahari., et al. "Effect of bleaching protocols on surface roughness and biofilm formation on silorane-based composite resin". (2019).

8. Kovacevic Pavicic., et al. "Changes in quality of life induced by tooth whitening are not influenced by global self-esteem: a randomized double-blind placebo-controlled trial". (2019).

9. Ito Y., et al. "Effect of pH conditioners on tooth bleaching". Clinical And Experimental Dental Research 5.3 (2019): 212-218.

Volume 3 Issue 10 October 2019

(C) All rights are reserved by Chiara Vagge. 